\title{
Uncertainty Modeling for Robustness Analysis of Failure Detection \& Accommodation Systems
}

\author{
Christine M. Belcastro \\ Mail Stop 161 \\ NASA Langley Research Center \\ Hampton, VA 23681-0001 \\ (757) 864-4035 \\ christine.m.belcastro@larc.nasa.gov
}

\section{Abstract}

Formal robustness analysis of failure detection and accommodation systems could play an important role in their validation and ultimate certification. However, formulation of linear fractional transformation (LFT) models can be very difficult for complex parameterdependent systems. This paper presents a matrix-based computational approach that can be implemented in software for obtaining LFT models of complex systems involving parametric uncertainties, faults, and failures. Several examples are presented, including: a modeling framework for performing robustness analyses of faulty uncertain systems and failure detection and accommodation systems.

\subsection{Introduction}

Certification of failure detection and accommodation systems for aircraft will require a comprehensive validation process (integrating analysis, simulation, and experimental methods) to ensure the safety and reliability of these systems. Robustness analysis for systems with structured uncertainty could play an important role in this process. Robustness is a key issue in the performance of failure detection and accommodation systems. Failure detection schemes can experience performance difficulties (such as false alarms) due to modeling uncertainties. Robustness of the control system can mask faults and failures and make the detection problem more difficult. It is fairly common for integration of failure detection and accommodation systems to be problematic if they're designed separately. Robustness analysis can also identify worst-case combinations of uncertainties, faults and failures for use in guided Monte Carlo simulation and/or experimental studies. For aircraft, robustness to nonlinear parameter variations over the flight envelope must also be considered.

Consider the generalized block diagram shown in Figure 1 for an integrated failure detection and accommodation system.

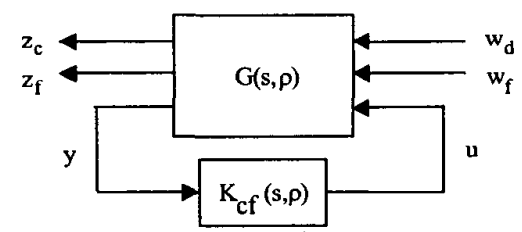

Figure 1. Generalized Block Diagram of the Integrated Failure Detection and Accommodation System

\author{
B.-C. Chang \\ ME\&M Dept \\ Drexel University \\ Philadelphia, PA 19104 \\ (215) 895-1790 \\ bchang@coe.drexel.edu
}

The block $G(s, \rho)$ represents the generalized plant model of the system as well as all of the associated weighting functions. The parameters $\rho$ contain a nominal component, $\rho_{o}$, and an uncertain component, $\delta$, as shown below for an additive representation.

$$
\rho=\rho_{0}+f(\delta)
$$

The term, $f(\delta)$, represents a function of the uncertain (and failure) parameters $\delta$. Closing the loop around $\mathrm{K}_{\mathrm{cf}}$ and separating the varying parameters yields the block diagram shown in Figure 2.

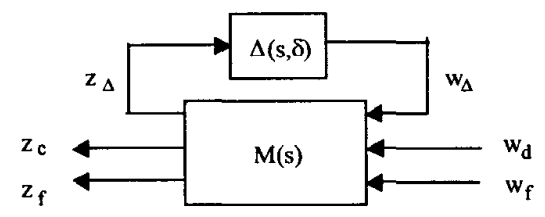

Figure 2. Generalized Block Diagram of the Closed-Loop Failure Detection and Accommodation System with Uncertainties

The $z_{c}$ and $z_{f}$ signals of represent controller and detection filter performance measures (respectively), and $w_{d}$ and $w_{f}$ represent exogenous signals associated with disturbances and failures (respectively). Note that robust performance in an $\mathrm{H}_{\infty}$ sense can be determined by closing the controller and filter signals around fictitious uncertainty blocks.

Block M(s) of Figure 2 contains nine transfer function matrices as defined below.

$$
\left[\begin{array}{l}
z_{\Delta} \\
z_{c} \\
z_{f}
\end{array}\right]=\left[\begin{array}{lll}
M_{z_{\Delta} w_{\Delta}} & M_{z_{\Delta} w_{d}} & M_{z_{\Delta} w_{f}} \\
M_{z_{c} w_{\Delta}} & M_{z_{c} w_{d}} & M_{z_{c} w_{f}} \\
M_{z_{f} w_{\Delta}} & M_{z_{f} w_{d}} & M_{z_{f} w_{f}}^{c}
\end{array}\right] *\left[\begin{array}{c}
w_{\Delta} \\
w_{d} \\
w_{f}
\end{array}\right]
$$

An interpretation of evaluating these transfer function matrices relative to the $\mathrm{H}_{\infty}$ norm is given in Table 1 , which is slightly more complete than that given in Ref. [1].

Table 1. Assessment of $M$ Relative to $\mathrm{H}_{\infty}$ Norm

\begin{tabular}{|l|l|}
\hline$\|\cdot\|_{\infty}$ & Interpretation \\
\hline$M_{z_{\Delta} w_{\Delta}}$ & $\begin{array}{l}\text { Measure of Stability Robustness Relative to the } \\
\text { Uncertainties in } \Delta(\mathrm{s}, \delta)\end{array}$ \\
\hline $\mathrm{M}_{z_{\mathrm{c}} w_{\Delta}}$ & $\begin{array}{l}\text { Measure of Control Performance Robustness } \\
\text { Relative to Uncertainties in } \Delta(\mathrm{s}, \delta)\end{array}$ \\
\hline
\end{tabular}

U.S. Government work not protected by U.S. Copyright 4776 


\begin{tabular}{|l|l|}
\hline$M_{z_{\mathrm{f}} w_{\Delta}}$ & $\begin{array}{l}\text { Measure of Robustness of Detection Filter } \\
\text { Relative to Uncertainties in } \Delta(\mathrm{s}, \delta)\end{array}$ \\
\hline $\mathrm{M}_{z_{\mathrm{c}} w_{\mathrm{d}}}$ & $\begin{array}{l}\text { Measure of Disturbance Rejection in Control } \\
\text { Performance Relative to Exogenous Inputs }\end{array}$ \\
\hline $\mathrm{M}_{z_{\mathrm{f}} w_{\mathrm{d}}}$ & $\begin{array}{l}\text { Measure of Disturbance Rejection in Detection } \\
\text { Filter - Disturbances Are Not Readily Detected } \\
\text { as Faults (Reduced False Alarms) }\end{array}$ \\
\hline $\mathrm{M}_{z_{\mathrm{c}} w_{\mathrm{f}}}$ & Effect of Faults on Control Performance \\
\hline $\mathrm{M}_{z_{\mathrm{f}} w_{\mathrm{f}}}$ & $\begin{array}{l}\text { Measure of Effectiveness of Detection Filter in } \\
\text { Providing an Estimate of Actuator and Sensor } \\
\text { Failures }\end{array}$ \\
\hline $\mathrm{M}_{z_{\Delta} w_{\mathrm{d}}}$ & Effect of Disturbances on Uncertainties \\
\hline $\mathrm{M}_{z_{\Delta} w_{\mathrm{f}}}$ & Effect of Failures on Uncertainties \\
\hline
\end{tabular}

Note that all of the interconnection matrices in $M$ associated with $\Delta(s, \delta)$ must be determined in order to formulate the generalized block diagram of Figure 2. This requires formulation of an LFT model (see Reference [2]), which can be extremely difficult and time consuming especially for parametric uncertainties (see References [3] [11]). In fact, the difficulty in formulating the uncertainty model in LFT form is a key impediment to performing robustness analyses for these systems. Section 2 presents a matrix-based computational approach for obtaining LFT models for complex systems involving parametric uncertainties. Several examples are presented in Section 3, to demonstrate the modeling approach and provide a more detailed modeling framework for analysis. Section 4 presents some concluding remarks.

\subsection{Parametric LFT Modeling}

The LFT model to be solved in this section is depicted in Figure 3.

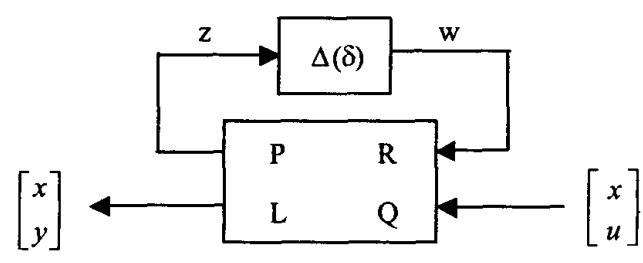

Figure 3. Block Diagram of LFT Modeling Problem

The matrix $\Delta(\delta)$ contains the system uncertainties, and can be represented as follows for parametric uncertainties.

$$
\begin{gathered}
\Delta(\delta)=\operatorname{diag}\left[\delta_{1} \mathrm{I}_{\mathrm{n}_{1}}, \delta_{2} \mathrm{I}_{\mathrm{n}_{2}}, \ldots, \delta_{\left.\mathrm{m} \mathrm{I}_{\mathrm{m}}\right]}\right] \\
\operatorname{dim}[\Delta(\delta)]=\mathrm{n}=\sum_{\mathrm{i}=1}^{\mathrm{m}} \mathrm{n}_{\mathrm{i}} \\
=\left[{ }_{1}, 2, \cdots, \mathrm{m}_{\mathrm{m}}\right] \in \mathrm{R}^{\mathrm{m}}
\end{gathered}
$$

The LFT equation associated with Figure 3 is given below.

$$
\begin{aligned}
& \mathrm{S}(\delta)=\mathrm{L}(\mathrm{I}-\Delta \mathrm{P})^{-1} \Delta \mathrm{R}+\mathrm{Q} \\
& \Rightarrow \mathrm{S}(\delta)=\mathrm{S}_{\Delta}(\delta)+\mathrm{So}
\end{aligned}
$$

The matrix $S(\delta)$ is a compact representation of the system model. The matrix $Q$ represents the nominal system model. The interconnection matrices $\mathrm{P}, \mathrm{R}$, and $\mathrm{L}$ are to be determined for the uncertain component of $S$ using the following equation.

$$
\mathrm{S}_{\Delta}(\delta)=\mathrm{L}(\mathrm{I}-\Delta \mathrm{P})^{-1} \Delta \mathrm{R}
$$

Note that $S_{\Delta}(\delta)$ contains given system matrices which are functionally dependent on the parameters $\delta$. A solution for equation (5) is summarized below for $S_{\Delta}(\delta)$ formulated as a multivariate polynomial matrix function of $\delta$. However, it should be noted that multivariate rational functions can also be formulated and solved using this approach (see Ref. [6]).

Equation (5) can be solved for multivariate polynomial problems by replacing the matrix inversion with a finite series expansion and a nilpotency condition,

$$
\begin{gathered}
\mathrm{S}_{\Delta}(\delta)=\mathrm{L} \Delta \mathrm{R}+\mathrm{L}\left[\Delta \mathrm{P}+(\Delta \mathrm{P})^{2}+\ldots+(\Delta \mathrm{P})^{\mathrm{r}}\right] \Delta \mathrm{R} \\
(\Delta \mathrm{P})^{r+1}=0
\end{gathered}
$$

where $r$ is determined by the degree of the largest nonzero term in $\mathrm{S}_{\Delta}(\delta)$. An expanded definition of $\mathrm{P}, \mathrm{R}$, and $\mathrm{L}$ containing matrix partitions associated with the $\delta_{\mathrm{i}} \mathrm{I}_{\mathrm{n}_{\mathrm{i}}}$ blocks of $\Delta$ given in Eqn. (2) are defined for $i, j=1,2, \ldots, m$.

$$
\begin{gathered}
\mathbf{L}=\left[\begin{array}{llll}
\mathbf{L}_{1} & \mathbf{L}_{2} & \cdots & \mathbf{L}_{m}
\end{array}\right] \\
\mathbf{R}=\left[\begin{array}{c}
\mathbf{R}_{1} \\
\mathbf{R}_{2} \\
\vdots \\
\mathbf{R}_{m}
\end{array}\right], \quad \mathbf{P}=\left[\begin{array}{cccc}
\mathbf{P}_{11} & \mathbf{P}_{12} & \ldots & \mathbf{P}_{1 m} \\
0 & \mathbf{P}_{22} & \cdots & \mathbf{P}_{2 m} \\
0 & 0 & \ddots & \vdots \\
0 & 0 & 0 & \mathbf{P}_{m m}
\end{array}\right]
\end{gathered}
$$

where:

$$
P_{i j} \in R^{n_{i} n_{j}}, \quad L_{i} \in R^{n_{\text {rows }} n_{i}}, R_{i} \in R^{n_{i} n^{n}} \text { cols }
$$

and each $P$ main-diagonal block is nilpotent of index $\eta_{i}$ :

$$
\begin{aligned}
& \left(P_{i i}\right)^{\eta_{i}}=0, \quad \eta_{i} \quad n_{i}, i=1,2, \ldots, m \\
& \eta_{i}=\text { maximum degree of } \delta_{i} \text { in } S_{\Delta}(\delta)
\end{aligned}
$$

The block-triangular structure of $P$ is sufficient but not necessary for nilpotency, and other special structures can also be found. Solution of Eqn. (6) for the matrices L, P, R and $\Delta(\delta)$ can then be reduced to solving the following set of equations.

Linear $\delta_{\mathrm{i}}$ Terms:

$$
\mathrm{L}_{\mathrm{i}} \mathrm{R}_{\mathrm{i}_{\mathrm{i}}}=\mathrm{S}_{\Delta_{\delta_{\mathrm{i}}}}, \mathrm{i}=1,2, \ldots, \mathrm{m}
$$


$\xi^{\text {th }}$-Degree $\delta$; Terms:

$\mathrm{L}_{\mathrm{i}}\left(\mathrm{P}_{\mathrm{ii}}\right){ }^{-1} \mathrm{R}_{\mathrm{i}} \mathrm{S}_{{ }_{\left(\delta_{\mathrm{i}}\right)}}, \mathrm{i}=1,2, \ldots, \mathrm{m} ; \xi=1,2, \ldots, \eta_{\mathrm{i}}$

Crossterms:

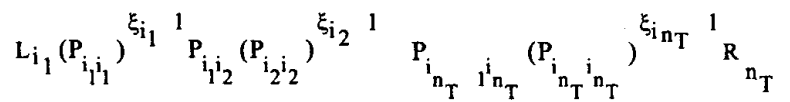$$
{ }^{S} \Delta_{\left(\delta_{i_{1}}\right)}{ }^{i_{1}}\left(\delta_{i_{2}}\right){ }^{i} 2 \ldots\left(\delta_{i_{n_{T}}}\right){ }^{i n_{T}}
$$

where:

$$
\begin{gathered}
\xi=\xi_{i_{1}}+\xi_{i_{2}}+\ldots+\xi_{i_{T}} \\
i_{1}=1,2, \ldots, m-\left(n_{T}-1\right) \\
i_{2}=i_{1}+1, i_{1}+2, \ldots, m-\left(n_{T}-2\right) \\
i_{n_{T}}=i_{1}+\left(n_{T}-1\right), \ldots, m
\end{gathered}
$$

$\mathrm{n}_{\mathrm{T}}=$ number of parameters in the crossterm $\mathrm{m}$

Note that the $S_{\Delta}$ terms on the right-hand side of Eqns. (11) through (13) are the known constant matrix coefficients associated with the indicated parameter terms in $S_{\Delta}(\delta)$. Moreover, depending on the number of parameters and the degree of each appearing in $S_{\Delta}(\delta)$, there can be literally hundreds of $S_{\Delta}$ coefficient terms and coupled matrix equations to be solved (or more). Moreover, satisfying these equations as simultaneously as possible to take advantage of any common structure (and reduce the resulting model dimension) while satisfying the nilpotency condition of Eqn. (7) is highly nontrivial.

\subsection{Solution of $\mathrm{L}, \mathrm{R}$, and Main-Diagonal Blocks of $\mathrm{P}$ :}

A solution for this part of the problem is given in Refs. [7] and [10], but is summarized here for completeness. The blocks of $L$ and $R$, and the main-diagonal blocks of $P$ are solved simultaneously for each uncertain parameter $\delta_{i}$ using the linear and $\xi^{\text {th }}$-degree $\delta_{i}$ terms. The solution is accomplished such that the resulting main-diagonal blocks of $P$ are nilpotent with the appropriate index of nilpotency equal to the maximum parameter degree in $S_{\Delta}(\delta)$. This solution is accomplished numerically with a matrix singular value decomposition (svd) by recognizing that this part of the problem is equivalent to a 1-D state-space (minimal) realization problem and by appropriately defining the equivalent block Hankel matrices. The solution is accomplished for each $\delta_{j}$ parameter as shown by the following theorem.

\section{Theorem}

Consider the linear and $\zeta^{\text {th }}$-degree $\delta_{\mathrm{i}}$ terms of $\mathrm{S}_{\Delta}(\delta)$, which can be expanded as follows

$$
\mathrm{S}_{\Delta_{L, \zeta}}(\delta)=\left[\mathrm{S}_{\Delta_{\delta_{i}}}\right] \delta_{i}+\left[\mathrm{S}_{\Delta_{\delta_{i}^{2}}}\right] \delta_{i}^{2}+\ldots+\left[\mathrm{S}_{\Delta_{\delta_{i}} \eta_{i}}\right] \delta_{i}^{\eta_{i}}
$$

and use the constant coefficient matrices of Eqn. (14) to form the block Hankel matrices defined below

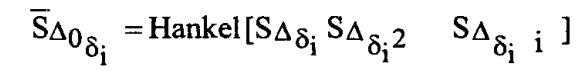

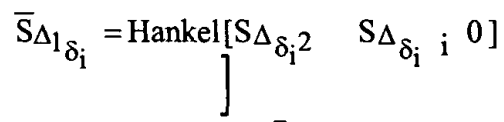

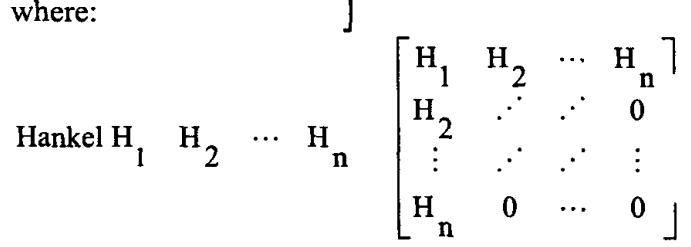

Eqn. (16) can be constructed from (15) by shifting each block row up and filling in the bottom block row with zero blocks. Define the matrix svd of Eqn. (15) as follows.

$$
\begin{gathered}
\bar{s}_{\Delta_{0}}=U_{i} \Sigma_{i} V_{i}{ }^{T} \\
=\left(U_{i} \Sigma_{i}{ }^{1 / 2}\right)\left(i_{i}^{1 / 2} V_{i}{ }^{T}\right) \bar{L}_{i} \bar{R}_{i}
\end{gathered}
$$

where:

$$
\operatorname{rank}\left(\overline{\mathrm{S}}_{\Delta_{0} \delta_{i}}\right)=\operatorname{rank}\left(\bar{L}_{i}\right)=\operatorname{rank}\left(\bar{R}_{i}\right)
$$

Then, $\left\{\mathbf{P}_{\mathrm{ii}}, \mathrm{R}_{\mathrm{i}}\right\}$ is controllable and $\left\{\mathrm{L}_{\mathrm{i}}, \mathbf{P}_{\mathrm{ii}}\right\}$ is observable, and the matrices $\boldsymbol{F}_{i}, R_{i}$, and $P_{i i}$ form an irreducible realization of $S_{\Delta_{L, \zeta}}(\delta)$ as defined by Eqn. (14), where:

$$
\begin{gathered}
\mathrm{L}_{\mathrm{i}}=\mathrm{I}_{\mathrm{n}_{\text {rows }}} 0 \overline{\mathrm{L}}_{\mathrm{i}} \quad, \quad \mathrm{R}_{\mathrm{i}}=\overline{\mathrm{R}}_{\mathrm{i}}\left[\begin{array}{c}
\mathrm{I}_{\mathrm{n}_{\text {cols }}} \\
0
\end{array}\right] \\
\mathrm{P}_{\mathrm{ii}} \quad\left(\overline{\mathrm{L}}_{\mathrm{i}}\right)^{\dagger} \overline{\mathrm{S}}_{\Delta_{1_{1}}}\left(\overline{\mathrm{R}}_{\mathrm{i}}\right)^{\dagger}
\end{gathered}
$$

and the notation $(\mathrm{A})^{\dagger}$ designates the pseudoinverse of matrix A. The $P_{i i}$ matrix is nilpotent with index $\eta_{i}$.

\subsection{Solution of P Off-Diagonal Blocks:}

The $\mathrm{P}$ off-diagonal blocks are each solved using the appropriate crossterms of $S_{\Delta}(\delta)$, as defined by Eqn. (13). The equation to be solved for each off-diagonal block of $P$ is a generalized linear matrix equation. The general form of the equation is given below for computing $P_{n j}$, where $n=1$, $2, \ldots, m-1$ and $j=n+1, n+2, \ldots, m$.

$$
\bar{A}_{n}{ }^{[n]} P_{n j} B_{j}=\bar{S}_{\Delta \delta n \delta j}{ }^{[n]}
$$

The matrices $\bar{A}_{n}{ }^{[n]}, B_{j}$, and $\bar{S}_{\Delta_{\delta n} \delta_{j}}{ }^{[n]}$ in Eqn. (21) are comprised of known matrices as well as matrices that have already been computed at this point in the solution process. The equations for Block Rows 1 and 2 are given below. 
Block Row 1:

$$
\bar{A}_{1}^{[1]} P_{1 j} B_{j}=\bar{S}_{\Delta}{ }_{1 j}^{[1]}
$$$$
\text { where: } \quad \overline{\mathbf{A}}_{1}^{[1]}=\left[\begin{array}{cc}
\mathbf{L}_{1} & \\
\mathbf{L}_{1} \mathbf{P}_{11} & \\
\vdots & \\
\mathbf{L}_{1} \mathbf{P}_{11} &
\end{array}\right]
$$

Block Row 2:

$$
\overline{\mathbf{A}}_{2}^{[2]} \mathrm{P}_{2 \mathrm{j}} \mathrm{B}_{\mathrm{j}}=\overline{\mathrm{S}}_{\Delta}{ }_{2 \mathrm{j}}^{[2]}
$$

where:

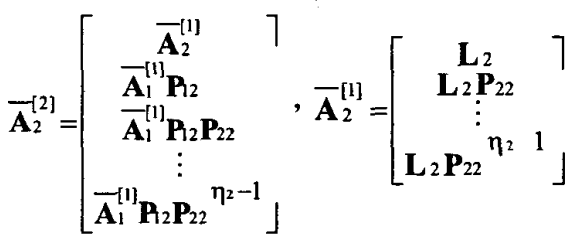

$B_{\mathrm{j}}$ Matrix Structure for Each Block Row:

$$
\left.\mathbf{B}_{j} \equiv \overline{\mathbf{R}}_{j}=\mathbf{R}_{j} \mathbf{P}_{j j} \mathbf{R}_{j} \cdots \mathbf{P}_{j j}{ }^{\eta j}{ }^{1} \mathbf{R}_{j}\right]
$$

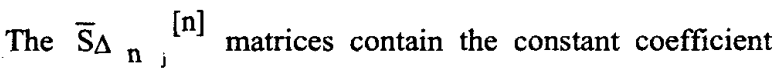
matrices associated with the cross-product terms being solved.

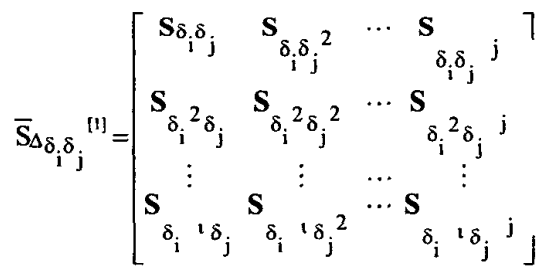

where: $i=1,2, \ldots, j-1, j=2,3, \ldots, m(i \neq j)$

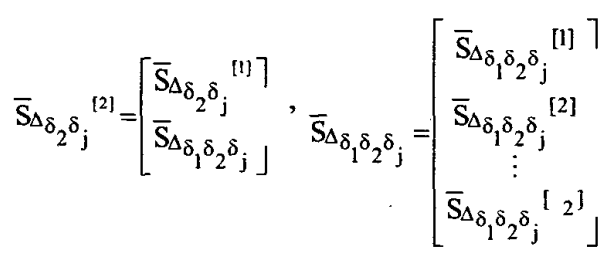

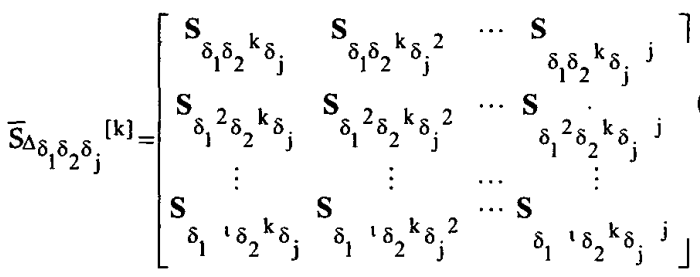

where: $\mathrm{k}=1,2, \ldots, \eta_{2}$
The off-diagonal block equations for Block Row 1, Eqns. (22), (24) and (25a) for $\mathrm{i}=1$, solve all pair-wise crossproduct terms associated with $\delta_{1} \delta_{j}$. The off-diagonal block equations for Block Row 2, Eqns. (23), (24), and (25) for $\mathrm{i}=2$, solve all pair-wise cross-product terms associated with $\delta_{2} \delta_{j}$, plus all triple terms involving $\delta_{1} \delta_{2} \delta_{j}$. The third block row equations (not shown) solve all pair-wise crossterms associated with $\delta_{3} \delta_{j}$, plus all triples associated with $\delta_{1} \delta_{3} \delta_{j}$ and $\delta_{2} \delta_{3} \delta_{j}$, plus all quadruple terms associated with $\delta_{1} \delta_{2} \delta_{3} \delta_{j}$. Thus, the equations for the $i^{\text {th }}$ block row solves the pair-wise crossterms associated with $\delta_{\mathrm{i}} \delta_{\mathrm{j}}$, plus all combinations of crossterms involving $\delta_{1}, \delta_{2}, \ldots, \delta_{i}$ and $\delta_{j}$. Note that the solutions include all $\mathrm{n}^{\text {th }}$-degree terms. This is accomplished by the main-diagonal blocks of $\mathrm{P}\left(\mathrm{P}_{\mathrm{ii}}\right)$ raised to various powers up to $\eta_{i}-1$, as defined by Eqn. (10b), appearing in the $\mathrm{A}$ and $\mathrm{B}$ matrices of Eqn. (21). The general expressions for Eqn. (21) are given in Ref. [10], but details of an approach for solving them are given below.

Eqn. (21) is a generalized linear matrix equation of the form

$$
\mathrm{AXB}=\mathrm{C}
$$

where $\mathrm{A}, \mathrm{B}$, and $\mathrm{C}$ are known constant matrices. Thus, solution of the off-diagonal blocks of $\mathrm{P}$ can be reduced to solving matrix equations of the form of Eqn. (26), which requires satisfaction of the following rank conditions.

$$
\operatorname{rank}[A \quad C]=\operatorname{rank}[A], \operatorname{rank}\left[B^{T} C^{T}\right]^{T}=\operatorname{rank}[B]
$$

Satisfaction of the column rank condition (first) and row rank condition (second) of Eqn. (27) is accomplished through augmentation of the dimension of the appropriate parameters, $\delta_{i}$, in $\Delta$, which translates to an augmentation of the associated partitions of $\mathrm{L}, \mathrm{R}$, and $\mathrm{P}$ comprising the $\mathrm{A}$ and B matrices in Eqn. (26). This is a nontrivial task, because columns or rows of $\mathrm{C}$ cannot simply be appended to $\mathrm{A}$ and $\mathrm{B}$. For the column rank condition, the structure of A becomes more complicated for successive block rows of $P$, for higher numbers of parameters, and for higher parameter degrees. For the row rank condition, the $B$ matrix structure is fixed, but is more complicated for higher parameter degrees.

Another complication to performing the augmentation is that in augmenting the underlying $L, R$, and $P$ matrix partitions of $\mathrm{A}$ and $\mathrm{B}$, the previously obtained solutions involving these partitions must be retained, as well as the nilpotency (with the correct nilpotency index) of the maindiagonal blocks of $P$. Moreover, the augmentation process must be general and implementable for any number of parameters and for any parameter degree.

The approach taken in this paper to solve the above augmentation problem is based on utilizing the structure of the $A$ and $B$ matrices (i.e., involving successive powers of nilpotent matrices), and allows an arbitrary augmentation to be performed independently of the coefficient matrices in C. This enables the satisfaction of the rank conditions of Eqn. (27) for any problem. The basic result is given in the 
following Lemma, which is an extension of a Theorem by Halmos in Ref. [13].

\section{Lemma}

Let $N \in R^{n \times n}$ be a nilpotent matrix of index $q$, and $M \in$ $\mathrm{R}^{\mathrm{nxm}}(\mathrm{n}>\mathrm{m})$ be an arbitrary rank $m$ matrix such that $\operatorname{rank}\left(\mathrm{N}^{\mathrm{q}-1} \mathrm{M}\right)=\mathrm{m}$. Then, the columns of $\mathrm{M}, \mathrm{NM}, \ldots, \mathrm{N}^{\mathrm{q}-1} \mathrm{M}$ are linearly independent; i.e., for $\mathrm{n} \geq \mathrm{qm}$ :

$$
\begin{aligned}
& \qquad \operatorname{Rank}\left[M, N M, \ldots, N^{q-1} M\right]=q m \\
& \text { where }\left[M, N M, \ldots, N^{q-1} M\right] \in R^{n x q m} .
\end{aligned}
$$

This lemma directly applies to the $\mathrm{B}$ matrix given by Eqn. (24); its dual (obtained by taking transposes) can be directly applied to the A matrix for block row 1, as given by Eqn. (22b). However, it can be shown that the lemma can also be applied to the off-diagonal block solution for any block row by reformulating the associated A matrix to be in the form of Eqn. (28). Thus, arbitrary columns can be added to the columns of $L_{i}$, and arbitrary rows can be added to the rows of $R_{j}$ during the augmentation process. Moreover, an arbitrary nilpotent augmentation of the correct nilpotency index can be added to the main diagonal block partitions. The augmented matrices become:

$$
\left.\mathbf{L}_{\mathbf{i}_{\text {aug }}} \quad \mathbf{L}_{\mathbf{i}} \hat{\mathbf{L}}_{\mathrm{i}}\right], \mathbf{R}_{\mathrm{i}_{\text {aug }}}\left[\begin{array}{c}
\mathbf{R}_{\mathrm{i}} \\
\hat{\mathbf{R}}_{\mathrm{i}}
\end{array}\right], \mathbf{P}_{\mathrm{ii}} \quad\left[\begin{array}{cc}
\mathbf{P}_{\mathrm{ii}} & 0 \\
\mathbf{0} & \hat{\mathbf{P}}_{\mathrm{ii}}
\end{array}\right]
$$

where $\hat{\mathbf{P}}_{\mathrm{ii}}$ is obtained using the general description for a nilpotent matrix given in Ref. [14].

The general process is to augment $\mathrm{L}_{\mathrm{i}}$ and $\mathrm{P}_{\mathrm{ii}}$ to satisfy the column rank condition for block row $i$, and to augment $R_{j}$ and $P_{j j}$ to satisfy the row rank condition for block column j. In order to retain previous solutions and to permit arbitrary augmentations to previously computed $P_{i j}$ matrices, $R_{i}\left(L_{j}\right)$ is augmented with zero rows (columns) when $L_{i}$ and $P_{i i}\left(R_{j}\right.$ and $\left.P_{j j}\right)$ are augmented to satisfy the column (row) rank condition.

The augmentation scheme described above has been implemented as part of a software tool for parametric LFT modeling. The tool has been developed for general problems involving $\mathrm{m}$ parameters each raised to any maximum degree, plus all possible cross-product terms. This tool is currently being tested and refined. Preliminary testing of $\sim 60$ randomly generated problems indicates that the tool is working correctly. Current refinement efforts are focused on ensuring a low-order model results for all problems.

\subsection{Full P- $\Delta$ Model Solution:}

Once the $L_{i}, R_{i}, P_{i i}$, and $P_{i j}$ matrices for each parameter have been determined, the full solution is assembled. This is a simple matter of collecting the matrix partitions together into the full $\mathrm{L}, \mathrm{R}$, and $\mathbf{P}$ matrices defined in Eqn. (8). The $\Delta$ matrix is also known and given by Eqn. (2), where the number of repetitions for each parameter, $n_{i}$, was determined in solving the $L_{i}, R_{i}, P_{i i}$, and $P_{i j}$ matrices.

\subsection{Modeling Examples}

Several examples using the LFT modeling approach have been presented in References [7] and [12]. This section contains a more complicated LFT modeling example, an example modeling framework for robustness analysis of failure detection and accommodation systems, and a faulty uncertain aircraft system model (using the model presented in Ref. [12]).

\subsection{LFT Modeling Example}

In order to test the augmentation approach presented in Section 2 for solution of the off-diagonal blocks of $P$, a complicated example problem was generated by extending the multivariate quadratic matrix problem presented in Ref. [7]. The original problem involved 3 uncertain parameters with maximum degree 2 , coefficient matrices of dimension $3 \times 6$, and 6 non-zero matrix coefficients (out of a total of 26 possible terms). The extended example presented here involves the same 3 uncertain parameters but with the first having a maximum degree of 3 , the same coefficient matrix dimensions, and 32 non-zero matrix coefficients (out of a total of 35 possible terms). The LFT modeling approach presented in Section 3 was applied to] this example, and the resulting model is summarized below.

$$
\begin{aligned}
& \mathbf{P}=\left[\begin{array}{ccc}
\mathbf{P}_{x x} & \mathbf{P}_{x y} & \mathbf{P}_{x z} \\
0 & \mathbf{P}_{y y} & \mathbf{P}_{y z} \\
0 & 0 & \mathbf{P}_{z z}
\end{array}\right], \quad \mathbf{L} \quad \mathbf{L}_{x} \mathbf{L}_{y} \mathbf{L}_{z} \quad, \quad \mathbf{R}=\left[\begin{array}{l}
\mathbf{R}_{x} \\
\mathbf{R} y \\
\mathbf{R}_{z}
\end{array}\right] \\
& \Delta=\left[\begin{array}{ccc}
\delta_{x} \mathrm{I}_{n_{x}} & 0 & 0 \\
0 & \delta_{y} \mathrm{I}_{n y} & 0 \\
0 & 0 & \delta_{z} \mathrm{I}_{n_{z}}
\end{array}\right]
\end{aligned}
$$

where:

$$
\mathrm{n}_{\mathrm{x}}=9, \mathrm{n}_{\mathrm{y}}=24, \mathrm{n}_{\mathrm{z}}=12 \Rightarrow \mathrm{n}_{\Delta}=45
$$

Due to the matrix dimensions and limited space, the numerical values of the above matrix partitions are not included. However, the resulting model is correct, is irreducible when each parameter is tested as a 1dimensional system, and the nilpotency conditions are satisfied to the proper index.

\subsection{Example Modeling Framework for Analysis of Failure Detection \& Accommodation Systems}

Figure 4 presents an example modeling framework that illustrates the formulation (at a more detailed level) of an analysis model with parametric uncertainty that can be translated to the general model depicted in Figure 2. This example is an extension of the modeling approach presented in Refs. [1] and [15].

The plant model contains two sources of uncertainty: operational variations $(\Delta)$ and component uncertainty $\left(\Delta_{c}\right)$. The operational variations can include nonlinear parameter variations, as described in Ref. [12]. The component uncertainty can include variations in the underlying aerodynamic coefficients due to modeling uncertainty or to damage or icing, as well as variations to underlying 
subsystem components (e.g., sensors and actuators) due to subcomponent uncertainties or faults and failures (e.g., in the sensor and actuator dynamic models). The sensor and actuator blocks shown in Figure 4 represent sensor and actuator faults (excluding dynamics, which is included in the plant model), as described below.

$$
\begin{aligned}
& f_{s}=S_{s} \Delta_{s} y_{R}+d_{s} \\
& f_{a}=S_{a} \Delta_{a} u+d_{a}
\end{aligned}
$$

The $S_{\mathrm{S}}$ and $\mathrm{S}_{\mathrm{a}}$ matrices in Eqns. (30) represent scaling matrices. The da and ds vectors represent persistent disturbance signals, such as stuck actuators and sensor biases. These vectors are contained in the $\mathrm{du}$ and $\mathrm{dy}$ vectors of Figure 4, which can also contain other sources of disturbance such as turbulence and sensor noise, respectively.

The controller / filter block represents the failure detection filter and control accommodation system. The associated uncertainties $\left(\Delta_{\mathrm{cf}}\right)$ can represent controller gain scheduling (e.g., over the plant operational envelope) and/or reconfiguration under faults and failures, as well as any uncertainties and/or parameter dependence of the detection filter.

An example detection filter and controller performance specification is also given in Figure 4 (excluding frequency weighting matrices). The detection filter performance measure is defined as the sensor and actuator failure estimation error, given below.

$$
u f=\left[\begin{array}{l}
\hat{f a} \\
\hat{f s}\rfloor
\end{array}\right],-z f=\left[\begin{array}{l}
f a \\
f s
\end{array}\right] \text { uf }
$$

The controller performance measure for this example is defined as the error between the actual and some desired dynamic response. Uncertainties shown in Figure 4 for the model of the desired dynamic response were included to present variations in the desired dynamics under faults and failures. That is, the plant may not be able to have the same dynamic response under faults or damage as under nominal conditions.

The analysis model of Figure 4 can be easily translated into the form of Figure 2 by pulling the $\Delta_{i}$ blocks associated with each subsystem into a single $\Delta$ block, and by deriving the partitions of $M$. Note that the LFT models for the uncertain subsystems can be determined independently, as shown in Figure 4, or the LFT model can be formulated simultaneously for those subsystems that depend on the same parameters. An approach for doing this is discussed in Ref. [6] for rational problems.

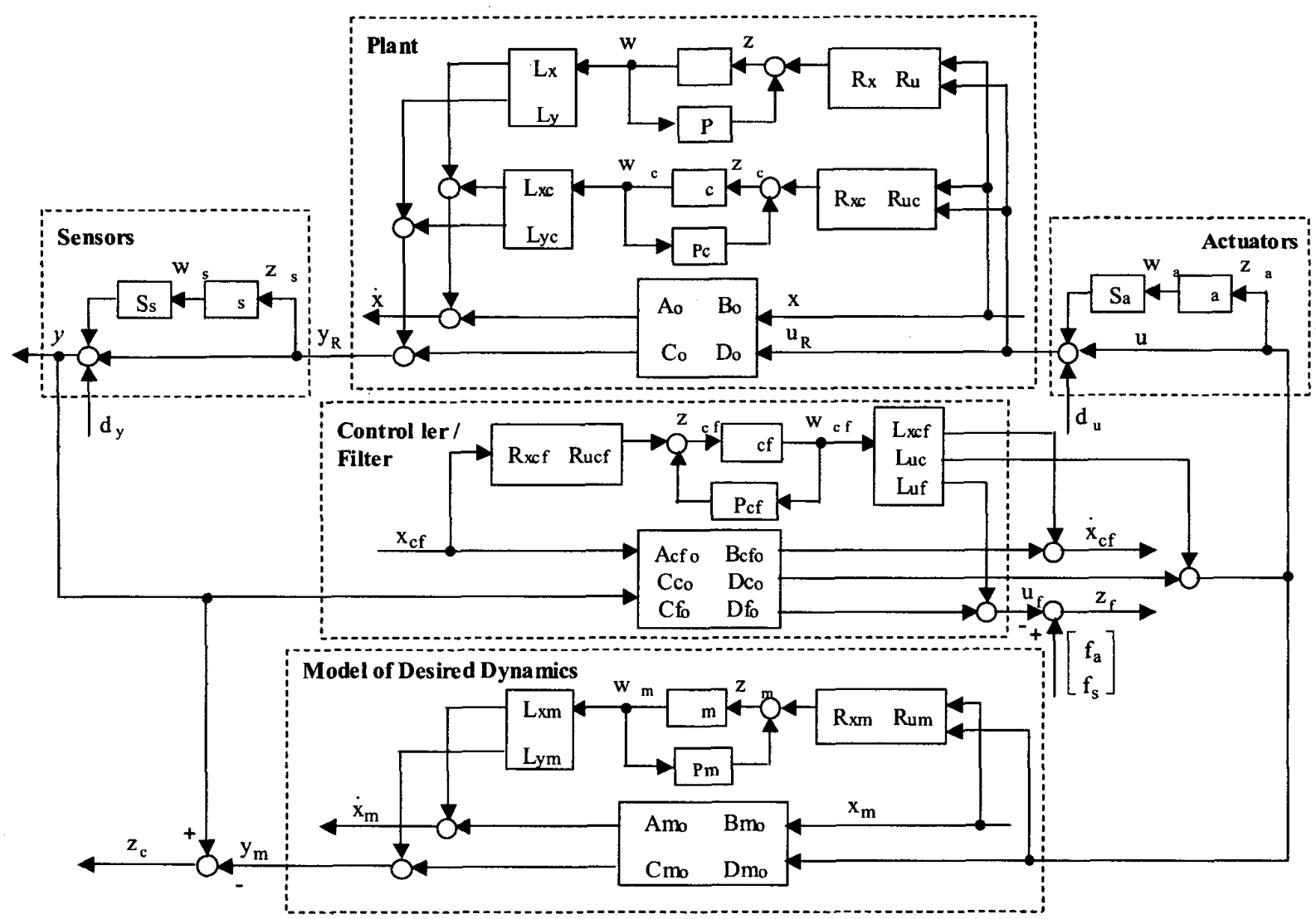

Figure 4. Example Modeling Framework for Robustness Analysis 


\subsection{Example Faulty Uncertain F-16 Aircraft Model}

Consider the open-loop faulty uncertain system model components of Figure 4, and the LFT model of the vehicle short period mode given in Ref. [12]. The F-16 parametric LFT model was based on a quasi-LPV (linear parameter varying) model with angle of attack $(\alpha)$ and longitudinal center of gravity $\left(\mathrm{x}_{\mathrm{cg}}\right)$ as the uncertain parameters. The quasi-LPV model was obtained over the $\alpha-x_{\mathrm{cg}}$ flight envelope of the vehicle. The variations in $\alpha$ were nonlinear (including up to $4^{\text {th }}$-degree terms) and are representative of a scheduling parameter. Variations in $x_{c g}$ were linear and are representative of uncertainties due to damage and/or store release. Cross-product terms were also included in the model. The resulting LFT model was of dimension 10, with 7 repetitions for $\delta_{\alpha}$ and 3 for $\delta_{\mathrm{x}_{\mathrm{cg}}}$.

Let the component uncertainty of Figure 4 . be comprised of additive linear variations up to $40 \%$ of the maximum nominal values of the $\mathrm{A}$ and $\mathrm{B}$ matrix elements for the F-16 due to modeling uncertainties and/or damage. Then, the open-loop system model of the form of Figure 2 is given below in Figure 5 .

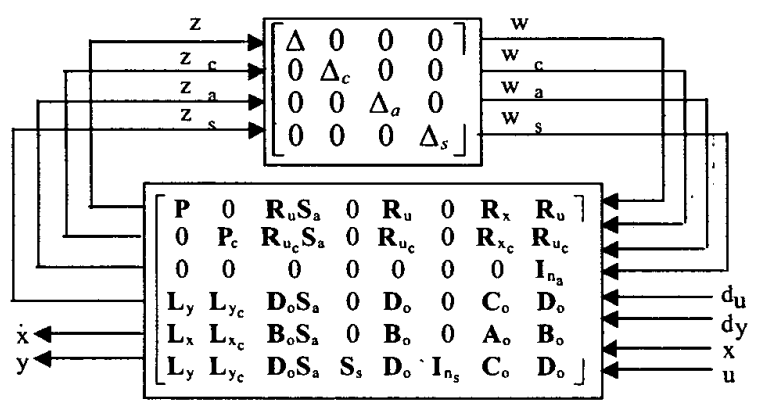

Figure 5. Generalized Block Diagram of the Faulty Uncertain F-16 System

The solutions for $P, L$, and $R$ are given in Ref. [12]: The matrix $P_{c}$ is a $6 \times 6$ zero matrix. The remaining component uncertainty block can be modeled as follows.

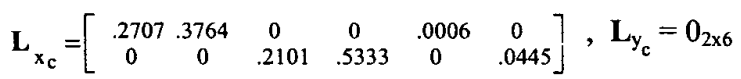

$$
\begin{aligned}
& \mathbf{R}_{\mathbf{x}_{\mathrm{c}}}=\left[\begin{array}{ll}
1 & 0 \\
0 & 1 \\
1 & 0 \\
0 & 1 \\
0 & 0 \\
0 & 0
\end{array}\right], \mathbf{R}_{\mathrm{u}_{\mathrm{c}}}=\left[\begin{array}{l}
0 \\
0 \\
0 \\
0 \\
1 \\
1
\end{array}\right] \\
& \Delta_{\mathrm{c}}=\operatorname{diag}\left[\begin{array}{llllll}
\delta_{\mathrm{a}_{11}} & \delta_{\mathrm{a}_{12}} & \delta_{\mathrm{a}_{21}} & \delta_{\mathrm{a}_{22}} & \delta_{\mathrm{b}_{11}} & \delta_{\mathrm{b}_{21}}
\end{array}\right]
\end{aligned}
$$

Assuming actuator and sensor scaling factors of $1, \mathrm{~S}_{\mathrm{s}}$ is a $2 \times 2$ identity matrix and $S_{a}$ is a unity scalar.

\subsection{Concluding Remarks}

This paper has presented a parametric LFT modeling method that can be applied to uncertainty faulty systems with nonlinear parameter variations. The approach is a matrix-based computational method that has been implemented in Matlab. The software is currently being tested and refined. The paper also presents an uncertainty modeling framework for performing robustness analyses of faulty uncertain systems and failure detection and accommodation systems. This modeling and analysis approach will be applied as part of a validation process being developed for potential certification of these systems.

\section{References}

[1] Stoustrup, Jakob, Grimble, M. F., and Niemann, Henrik: "Design of Integrated Systems for the Control and Detection of Actuator/Sensor Faults"; Sensor Review, Volumn 17, Number 2, pp. 138-149, 1997.

[2] Balas, Gary J., John C. Doyle, Keith Glover, Andy Packard, Roy Smith: $\mu$-Analysis and Synthesis Toolbox. The Math Works, Inc., MA, MUSYN, Inc., MN, 1991

[3] Morton, Blaise G., R. M. McAfoos: "A Mu-Test for Robustness Analysis of a Real-Parameter Variation Problem". Proc. of the ACC, pp. 135-138, 1985.

[4] Steinbuch, Maarten, et. al.: "Robustness Analysis for Real and Complex Perturbations Applied to an ElectroMechanical System". Proc. of the ACC, 1991.

[5] Lambrechts, Paul, et. al.: "Parametric Uncertainty Modeling using LFT's". Proc. of the ACC, Vol. 1, pp. 267-272, 1993.

[6] Belcastro, Christine M.: "Parametric Uncertainty Modeling: An Overview". Proc. of the ACC, 1998.

[7] Belcastro, Christine M. and Chang, B.-C.: "LFT Formulation for Multivariate Polynomial Problems". Proceedings of the ACC, 1998.

[8] Cockburn, Juan C: "Linear Fractional Representation of Systems with Rational Uncertainty". Proc. of the ACC, 1998.

[9] Beck, Carolyn \& D'Andrea, Raffaello: "Computa-tional Study and Comparisons of LFT Reducibility Methods". Proceedings of the ACC, 1998.

[10] Belcastro, Christine M.: "On the Numerical Formulation of Parametric Linear Fractional Transformation (LFT) Uncertainty Models for Multivariate Matrix Polynomial Problems". NASA TM-1998-206939, November 1998.

[11] Belcastro, Christine M., Lim, Kyong B. and Morelli, Eugene A.: "Computer-Aided Uncertainty Modeling of Nonlinear Parameter-Dependent Systems, Part I: Theoretical Overview". Proceedings of the CACSD, August 1999.

[12] Belcastro, Christine M., Lim, Kyong B. and Morelli, Eugene A.: "Computer-Aided Uncertainty Modeling of Nonlinear Parameter-Dependent Systems, Part II: F-16 Example". Proceedings of the CACSD, August 1999.

[13] Halmos, Paul R.: Finite-Dimensional Vector Spaces. Springer-Verlag New York, Inc., 1974

[14] Gantmacher, F. R.: The Theory of Matrices, Vol. I. Chelsea Publishing Company, New York, NY, 1959.

[15] Chen, Jie and Patton, R. J.: Robust Model-Based Fault Diagnosis for Dynamic Systems. Kluwer Academic Publishers, Norwell, MA, 1999. 